\title{
Deformation of finite morphisms and smoothing of ropes
}

\author{
Francisco Javier Gallego, Miguel González and Bangere P. Purnaprajna \\ Dedicated to David Eisenbud on his sixtieth birthday
}

\begin{abstract}
In this paper we prove that most ropes of arbitrary multiplicity supported on smooth curves can be smoothed. By a rope being smoothable we mean that the rope is the flat limit of a family of smooth, irreducible curves. To construct a smoothing, we connect, on the one hand, deformations of a finite morphism to projective space and, on the other hand, morphisms from a rope to projective space. We also prove a general result of independent interest, namely that finite covers onto smooth irreducible curves embedded in projective space can be deformed to a family of 1:1 maps. We apply our general theory to prove the smoothing of ropes of multiplicity 3 on $\mathbf{P}^{1}$. Even though this paper focuses on ropes of dimension 1, our method yields a general approach to deal with the smoothing of ropes of higher dimension.
\end{abstract}

\section{Introduction}

This paper contains two themes. The first part presents a general method to deal with ropes, their smoothings and their relation to deformations of finite morphisms. This method produces, among other things, two interesting and not previously known results. The first one is the fact that finite covers onto smooth irreducible curves embedded in projective space can be deformed to a family of $1: 1$ maps. The second one is the smoothing of most ropes of arbitrary multiplicity, supported on smooth curves. In the second part of the paper we carry out a detailed study of the projective embeddings of ropes of multiplicity 3 on $\mathbf{P}^{1}$ and we apply the general theory developed in the first part to prove that they are smoothable.

Let $Y$ be a smooth, irreducible projective curve. A rope $\tilde{Y}$ on $Y$ of multiplicity $m$ is a nowhere reduced scheme whose reduced structure is $Y$ and which locally looks like the first infinitesimal neighborhood of $Y$ inside the total space of a vector bundle of rank $m-1$. Such a scheme is projective and, even though singular everywhere, it is locally Cohen-Macaulay. The ideal sheaf of $Y$ inside of $\widetilde{Y}$ happens to be a locally free sheaf $\mathscr{E}$ of rank $m-1$, the so-called conormal bundle of $\widetilde{Y}$. The conormal bundle $\mathscr{E}$ is an important invariant of the rope and, together with $Y$, determines the arithmetic genus of $\widetilde{Y}$. On the other hand, fixing $Y$ and $\mathscr{E}$, there are in general many non-isomorphic rope structures $\widetilde{Y}$ on $Y$ having conormal bundle $\mathscr{E}$. Indeed, these rope structures are parametrized by $\operatorname{Ext}^{1}\left(\Omega_{Y}, \mathscr{E}\right)=H^{1}\left(\Omega_{Y}^{*} \otimes \mathscr{E}\right)$. For an introduction on ropes, the reader can look into [BE95], where Bayer and Eisenbud study at length ropes of multiplicity 2, the so-called ribbons.

Multiple structures appear in many contexts in algebraic geometry. For instance, the ubiquitously used so-called Ferrand-Szpiro doubling (that is, multiplicity 2 structures) is a very special case of

Received 10 October 2006, accepted in final form 24 August 2007, published online 14 March 2008.

2000 Mathematics Subject Classification 14H45, 14H10, 14B10, 13D10.

Keywords: degenerations of curves, multiple structures, deformations of morphisms.

This journal is (C) Foundation Compositio Mathematica 2008. 


\section{F. J. Gallego, M. González and B. P. Purnaprajna}

the structures of arbitrary multiplicity dealt with in this paper. The Ferrand-Szpiro doubling is used, mostly in an affine setting, in the theory of projective modules and in problems dealing with complete intersections, among other places. Projective multiple structures, which are more complicated, are the ones studied in this paper. The so-called canonical ribbons are another special case of these multiple structures and attracted considerable attention during the 1990s.

From a classical point of view, a geometer is primarily interested in smooth objects, or, at any rate, varieties. Thus, there are two questions one might reasonably ask, namely, whether ropes arise in a natural, uncontrived geometrical context, and what the connection of ropes to smooth varieties is. The answer to both matters is satisfactory. Precisely (see Theorems 1.1 and 2.4 and Corollary 2.7), a rope $\widetilde{Y}$ with non-negative arithmetic genus always arises as a flat limit of the images of a family of projective embeddings of smooth, irreducible curves, when those embeddings degenerate to $\pi$, provided that $\mathscr{E}$ satisfies the following condition:

$\mathscr{E}$ can be realized as the trace 0 module of a smooth, irreducible, finite cover $X \stackrel{\pi}{\rightarrow} Y$.

This answers right away the first question above: ropes appear indeed in a very natural and geometric way. It also answers the second question, for it implies that ropes are degenerations of smooth, irreducible curves, i.e. it shows that ropes can be smoothed.

Finding out that ropes are smoothable adds to our knowledge of the Hilbert schemes of curves. Precisely, we describe new points which lie in the boundary of components of the Hilbert scheme that parametrize smooth curves. This could shed light on whether Hilbert points of smooth curves can be connected by passing through Hilbert points of schemes without embedded points. Such is the case for curves in $\mathbf{P}^{3}$.

The way we prove the smoothing of ropes is also of independent interest. To be precise we prove the following important fact (see Theorem 1.1 and Corollary 1.2): finite covers onto smooth irreducible curves embedded in projective space can be deformed to a family of $1: 1$ maps.

The relation between ropes of multiplicity 2 and deformations of $2: 1$ morphisms has been studied previously. Fong (see [Fon93]) explored, using computational methods, the special case of canonical ribbons and its relation to the degeneration of canonical embeddings of smooth curves. Recently the second author (see [Gon06]) studied the case of ropes of multiplicity 2 on curves of arbitrary genus. In this context Theorem 1.1 is a more general result as it relates ropes of arbitrary multiplicity on curves and degenerations of embeddings to finite morphisms. In $\S 2$, this result is used to prove (see Theorem 2.4 and Corollary 2.7) that ropes with non-negative arithmetic genus and satisfying condition $(*)$ are smoothable.

In the second part of this paper ( $\S \S 3$ and 4 ) we focus on the study of ropes $\tilde{Y}$ of multiplicity 3 on $\mathbf{P}^{1}$, where the general theory can be made more explicit. The conormal bundle of $\widetilde{Y}$ is a vector bundle of rank 2, $\mathscr{E}=\mathscr{O}_{\mathbf{P}^{1}}(-a) \oplus \mathscr{O}_{\mathbf{P}^{1}}(-b)$. Since the arithmetic genus of $\widetilde{Y}$ is $a+b-2$, for a fixed arithmetic genus $p_{a}$ there exist ropes with different conormal bundles. Thus, for given $p_{a}$, there are different families of ropes which correspond to the non-negative number $n=|a-b|$. Ropes are more general when $n$ is smaller (see Proposition 4.4). This phenomenon is analogous to the one of trigonal curves, which are stratified in the moduli of curves by the so-called Maroni invariant. Because of this and by analogy with the Maroni invariant of smooth trigonal curves, we call $n$ the Maroni invariant of $\tilde{Y}$.

In $\S 3$ we study the morphisms and embeddings of ropes of multiplicity 3 on $\mathbf{P}^{1}$ to projective space. The main result in this regard is Theorem 3.2, which in particular gives the smallest projective space in which all ropes with fixed conormal bundle can be embedded. The uniform bound $N$ found depends on the arithmetic genus and on the Maroni invariant of the rope. We also study embeddings and morphisms from ropes to projective spaces of dimension smaller than $N$. 


\section{DEFORMATION OF FINITE MORPHISMS AND SMOOTHING OF ROPES}

Finally, in $\S 4$ we apply the results of $\S 2$ to smooth ropes of multiplicity 3 on $\mathbf{P}^{1}$, both as abstract schemes and as schemes embedded in projective space, in particular, the images of the embeddings studied in $\S 3$ (see Theorem 4.5). Knowledge of triple covers of $\mathbf{P}^{1}$ makes it possible to exactly characterize those ropes which satisfy $(*)$. These are ropes whose Maroni invariant is not too large compared to the genus. However, we are able to prove that not only the ropes that satisfy (*) are smoothable but also all other ropes with non-negative arithmetic genus. The reason for this is that ropes with higher Maroni invariant can be deformed to ropes with lower Maroni invariant (see Proposition 4.4).

The approach presented in this paper applies to other multiple structures such as ropes on surfaces. For instance, in [GGP07], we build on the methods developed here to show the smoothing of the so-called K3 carpets on Enriques surfaces. These ideas also shed light on the deformation of non-locally Cohen-Macaulay multiple structures. The ideas used here are quite novel in this context, comprising a combination of techniques from new deformation theory and suitable moduli arguments.

Convention. We work over an algebraically closed field $\mathbf{k}$ of characteristic 0 .

\section{Deformation of finite covers}

In this section we show that finite covers $X \stackrel{\pi}{\rightarrow} Y$ of curves can always be deformed to projective embeddings. More precisely, we see that $\pi$ can be realized as a morphism $\varphi$ from $X$ to a projective space $\mathbf{P}^{N}$, finite onto its image, which can be obtained as a degeneration of a family of embeddings of smooth curves into $\mathbf{P}^{N}$. This is essentially the content of Theorem 1.1 and Corollary 1.2. The proof of Theorem 1.1 builds upon the ideas of the proof of [Gon06, Theorem 5.1]. Theorem 1.1, however, is more general. The proof given here is also simpler and more transparent.

Theorem 1.1. Let $X \stackrel{\pi}{\rightarrow} Y$ be a cover of degree $m \geqslant 2$ between a smooth, irreducible, projective curve $X$ and a smooth, irreducible curve $Y$, embedded in $\mathbf{P}^{r}, r \geqslant 3$. Let $\mathscr{E}$ be the trace 0 module of $\pi$, i.e. let $\pi_{*} \mathscr{O}_{X}=\mathscr{O}_{Y} \oplus \mathscr{E}$. Let $\varphi$ denote the composition of $\pi$ with the inclusion of $Y$ in $\mathbf{P}^{r}$. Assume that

$$
h^{1}\left(\mathscr{O}_{Y}(1)\right)=0, \quad h^{1}\left(\mathscr{E} \otimes \mathscr{O}_{Y}(1)\right)=0 \quad \text { and } \quad h^{0}\left(\varphi^{*} \mathscr{O}_{Y}(1)\right) \geqslant r+1 .
$$

Let $\Delta=\operatorname{Spec} \mathbf{k}[\epsilon] / \epsilon^{2}$. Then for every first-order infinitesimal deformation

$$
\widetilde{X} \stackrel{\widetilde{\varphi}}{\rightarrow} \mathbf{P}_{\Delta}^{r}
$$

of $X \stackrel{\varphi}{\rightarrow} \mathbf{P}^{r}$, there exists a smooth irreducible family $\mathscr{X}$, proper and flat over a smooth pointed affine curve $(T, 0)$, and a $T$-morphism $\mathscr{X} \stackrel{\Phi}{\rightarrow} \mathbf{P}_{T}^{r}$ with the following features:

(1) the general fiber $\mathscr{X}_{t} \stackrel{\Phi_{t}}{\longrightarrow} \mathbf{P}^{r}, t \in T-0$, is a closed immersion of a smooth irreducible projective curve $\mathscr{X}_{t}$; and

(2) the fiber of $\mathscr{X} \stackrel{\Phi}{\rightarrow} \mathbf{P}_{T}^{r}$ over the tangent vector at $0 \in T$ is $\widetilde{X} \stackrel{\widetilde{\varphi}}{\longrightarrow} \mathbf{P}_{\Delta}^{r}$; in particular, the central fiber $\mathscr{X}_{0} \stackrel{\Phi_{0}}{\longrightarrow} \mathbf{P}^{r}$ is $X \stackrel{\varphi}{\longrightarrow} \mathbf{P}^{r}$.

Proof. Let us denote $d=-\operatorname{deg}\left(\bigwedge^{m-1} \mathscr{E}\right)$ and let $g$ be the genus of $Y$. Note that the genus of $X$ is $g_{X}=d+m g-m+1$. Let $L=\pi^{*} \mathscr{O}_{Y}(1)=\varphi^{*} \mathscr{O}_{\mathbf{P}^{r}}(1)$. The assumptions that $H^{1}\left(\mathscr{O}_{Y}(1)\right)=0$ and $H^{1}\left(\mathscr{E} \otimes \mathscr{O}_{Y}(1)\right)=0$ imply that $H^{1}(L)=0$. Moreover we have

$$
H^{0}(L)=H^{0}\left(\mathscr{O}_{Y}(1)\right) \oplus H^{0}\left(\mathscr{E} \otimes \mathscr{O}_{Y}(1)\right) .
$$

Let us denote

$$
\widetilde{L}=\widetilde{\varphi}^{*} \mathscr{O}_{\mathbf{P}_{\Delta}^{r}}(1)
$$




\section{F. J. Gallego, M. González and B. P. Purnaprajna}

Then the restriction of $\widetilde{L}$ to $X$ is $L$ and the $\Delta$-module $\Gamma(\widetilde{L})$ is free, of rank equal to $h^{0}(L)$, and $\Gamma(\widetilde{L}) \otimes k[\epsilon] / \epsilon k[\epsilon]=H^{0}(L)$.

We want to construct a family $\Phi$ of morphisms over $T$ such that its fiber over the tangent vector at 0 is $\widetilde{\varphi}$ and its general fiber is an embedding. We do this in two steps. First we construct a family $(\mathscr{X}, \mathscr{L})$, and second we construct $\Phi$ from this family.

Step 1. Construction of $(\mathscr{X}, \mathscr{L})$. We want to obtain a family $(\mathscr{X}, \mathscr{L})$, proper and flat over a smooth pointed affine curve $(T, 0)$, whose central fiber is $(X, L)$, whose restriction to the tangent vector to $T$ at 0 is $(\widetilde{X}, \widetilde{L})$ and whose general member $\left(\mathscr{X}_{t}, \mathscr{L}_{t}\right)$ consists of a smooth irreducible projective curve of genus $g_{X}$ and a very ample line bundle $\mathscr{L}_{t}$. If $g_{X} \leqslant 2$ then $L$ is very ample, since by hypothesis $\operatorname{deg} L \geqslant 6$. However, if $g_{X} \geqslant 3$, then $L$ is not necessarily very ample; therefore we distinguish two non-mutually exclusive cases, namely $g_{X} \geqslant 3$ and $L$ very ample.

Case $1.1\left(g_{X} \geqslant 3\right)$. Since we are not assuming $L$ to be very ample, the argument that follows will be rather technical, using the properties of the fine part of the moduli of curves and the result of Eisenbud and Harris [EH83, 5.1].

We consider $\widetilde{\omega}=\omega_{\widetilde{X} / \Delta}$ and $\widetilde{L}^{\prime}=\widetilde{L} \otimes \widetilde{\omega}^{\otimes n}$, where $n$ is large enough so that $L^{\prime}=L \otimes \omega_{X}^{\otimes n}$ is very ample, non-special and the complete linear series of $L^{\prime}$ defines an embedding $X \rightarrow X^{\prime} \subset \mathbf{P}^{r^{\prime}}$ that determines a smooth point $\left[X^{\prime}\right]$ in the corresponding Hilbert scheme. Let $H$ be the open, smooth and irreducible subset of this Hilbert scheme consisting of the points which correspond to smooth, irreducible, non-degenerate curves $C \subset \mathbf{P}^{r^{\prime}}$ of degree $d^{\prime}=m \operatorname{deg} \mathscr{O}_{Y}(1)+n\left(2 g_{X}-2\right)$ and genus $g_{X}$. Then $\left[X^{\prime}\right] \in H$. Since $n \gg 0$, for every such curve $C, \mathscr{O}_{C}(1)$ is non-special, the embedding of $C$ in $\mathbf{P}^{r^{\prime}}$ is defined by a complete series and defines a smooth point in its Hilbert scheme. Moreover, since $L^{\prime}$ is very ample and $H^{1}\left(L^{\prime}\right)=0, \widetilde{L}^{\prime}$ is very ample relative to $\Delta$ and the embedding $X \hookrightarrow \mathbf{P}^{r^{\prime}}$ extends to an embedding $\widetilde{X} \hookrightarrow \mathbf{P}_{\Delta}^{r^{\prime}}$. So the image $\widetilde{X}^{\prime}$ of $\widetilde{X} \hookrightarrow \mathbf{P}_{\Delta}^{r^{\prime}}$ is a flat family over $\Delta$ that corresponds to a tangent vector to $H$ at the Hilbert point $\left[X^{\prime}\right]$ of $X^{\prime}$. We can take the embedding $\widetilde{X} \hookrightarrow \mathbf{P}_{\Delta}^{r^{\prime}}$ so that this tangent vector is non-zero. Now, since $\left[X^{\prime}\right]$ is a smooth point in $H$, we can take a smooth irreducible affine curve $T$ in $H$ passing through $\left[X^{\prime}\right]$ with tangent direction the given tangent vector.

We can take the above curve in such a way that all its points except perhaps $\left[X^{\prime}\right]$ are placed in the open subset $U$ of $H$ constructed in the following way: $H$ admits a surjective morphism onto $\mathscr{P}_{d^{\prime}, g_{X}}$, the coarse moduli of pairs consisting of a curve of genus $g_{X}$ and a line bundle of degree $d^{\prime}$ on the curve. Denote $d_{1}=m \operatorname{deg} \mathscr{O}_{Y}(1)$ and consider also $\mathscr{P}_{d_{1}, g_{X}}$, fibered over $\mathscr{M}_{g_{X}}^{0}$, the fine part of the moduli space of curves of genus $g_{X}$. Let $\mathscr{C}^{\left(d_{1}\right)}$ be the scheme that represents the functor of relative effective Cartier divisors of relative degree $d_{1}$ on the universal curve $\mathscr{C}_{g_{X}}^{0} \rightarrow \mathscr{M}_{g_{X}}^{0}$ (see [Gro62, 4.1]). We have

$$
d_{1}-g_{X}=h^{0}(L)-1
$$

So from the assumptions $h^{0}(L) \geqslant r+1$ and $r \geqslant 3$ we have $d_{1}-g_{X} \geqslant 3$, in particular $d_{1} \geqslant g_{X}$. Therefore the morphism $\mathscr{C}^{\left(d_{1}\right)} \rightarrow \mathscr{P}_{d_{1}, g_{X}}$ is surjective. Denote $\mathscr{C}=\mathscr{C}_{g_{X}}^{0} \times{ }_{\mathscr{M}_{g_{X}}^{0}} \mathscr{C}^{\left(d_{1}\right)}$. Over $\mathscr{C}$ there is a universal effective relative Cartier divisor $\mathscr{D}$. Consider the line bundle $\mathscr{O}_{\mathscr{C}}(\mathscr{D})$ and let $\mathscr{C} \stackrel{q}{\rightarrow} \mathscr{C}^{\left(d_{1}\right)}$ be the (proper and flat) projection. Then, by the theorem of base change and cohomology, at a point $(C, D)$ of $\mathscr{C}^{\left(d_{1}\right)}$, consisting of a curve $C$ of genus $g_{X}$ and a non-special divisor $D$ of degree $d_{1}$ on $C$, the fiber of the coherent sheaf $R^{1} q_{*} \mathscr{O}_{\mathscr{C}}(\mathscr{D})$ is isomorphic to $H^{1}(C, D)$ and the same is true near $(C, D)$. So there is a non-empty open set $W_{1}$ in $\mathscr{C}^{\left(d_{1}\right)}$ formed by pairs consisting of a curve and a divisor whose associated line bundle is non-special. Furthermore, if we restrict to $W_{1}$, then the 


\section{DEFORMATION OF FINITE MORPHISMS AND SMOOTHING OF ROPES}

support of the cokernel of the natural map $q^{*} q_{*}\left(\mathscr{O}_{\mathscr{C}}(\mathscr{D})\right) \rightarrow \mathscr{O}_{\mathscr{C}}(\mathscr{D})$ is outside of the inverse image on an open set $W_{2} \subset W_{1}$.

So we obtain an open set $W_{2}$ on $\mathscr{C}^{\left(d_{1}\right)}$, formed by pairs consisting of a curve of genus $g_{X}$, and an effective divisor of degree $d_{1}$ whose associated line bundle is non-special and globally generated and such that its associated complete linear series has dimension $h^{0}(L)-1 \geqslant 3$. In [EH83, 5.1] it is proved that on a general smooth curve the general linear series of dimension at least 3 has no base points and its associated map to projective space is a closed immersion. Moreover, $\mathscr{C}^{\left(d_{1}\right)}$ is irreducible so $W_{1}$ dominates $\mathscr{M}_{g_{X}}^{0}$. Therefore, the set $W_{2}$ is non-empty, and shrinking $W_{2}$ so that $q_{*}\left(\mathscr{O}_{\mathscr{C}}(\mathscr{D})\right)$ is free of rank $h^{0}(L)$ on $W_{2}$, we have a $W_{2}$-morphism $\mathscr{C}_{g_{X}}^{0} \times_{\mathscr{M}_{g_{X}}^{0}} W_{2} \rightarrow \mathbf{P}_{W_{2}}^{h^{0}(L)-1}$. Now (see e.g. [Gro61, 4.6.7]), the points of $W_{2}$ such that the morphism induced on the fiber over the point is a closed immersion form an open set $W$ in $W_{2}$. So we obtain an open set $W$ in $\mathscr{C}^{\left(d_{1}\right)}$ formed by pairs consisting of a curve of genus $g_{X}$ and an effective divisor of degree $d_{1}$ whose associated line bundle is non-special and very ample. Also by [EH83, 5.1] , as $h^{0}(L)-1 \geqslant 3$, we see that the open set $W$ is non-empty. Now, since $\mathscr{C}^{\left(d_{1}\right)}$ is irreducible and $\mathscr{C}^{\left(d_{1}\right)} \rightarrow \mathscr{P}_{d_{1}, g_{X}}$ is surjective, we also obtain a non-empty open set in $\mathscr{P}_{d_{1}, g_{X}}$ formed by pairs consisting of a curve and a very ample non-special line bundle with as many global sections as $L$. Moreover, twisting by $\omega^{\otimes n}$ we have an isomorphism between $\mathscr{P}_{d_{1}, g_{X}}$ and $\mathscr{P}_{d^{\prime}, g_{X}}$. So we take the open set $U \subset H$, which is the inverse image of the considered open set in $\mathscr{P}_{d^{\prime}, g_{X}}$.

Let $0 \in T$ denote the point corresponding to $X^{\prime}$. Now, we take the curve $T$ so that $T-\{0\} \subset U$. Over the pointed affine curve $(T, 0)$ we have a proper flat polarized family $\left(\mathscr{X}, \mathscr{L}^{\prime}\right)$ whose fibers over 0 and over the tangent vector to $T$ at 0 are $\left(X, L^{\prime}\right)$ and $\left(\widetilde{X}, \widetilde{L}^{\prime}\right)$ respectively.

Now, twisting by $\omega_{\mathscr{X} / T}^{-n}$, we obtain a family $(\mathscr{X}, \mathscr{L})$, proper and flat over $T$, whose central fiber is $(X, L)$, whose restriction to the tangent vector to $T$ at 0 is $(\widetilde{X}, \widetilde{L})$ and whose general member $\left(\mathscr{X}_{t}, \mathscr{L}_{t}\right)$ consists of a smooth irreducible projective curve of genus $g_{X}$ and a very ample line bundle $\mathscr{L}_{t}$. Furthermore, the total family $\mathscr{X}$ is smooth and irreducible and $\mathscr{L}_{t}$ is non-special and has as many global sections as $L$ and degree $d_{1}=\operatorname{deg} L$.

Case 1.2 ( $L$ very ample). This happens when $g_{X} \leqslant 2$ or in most cases when $g_{X} \geqslant 3$. In this case the argument above can be drastically simplified. Because $L$ is very ample and non-special, its complete linear series defines an embedding $X \rightarrow X^{\prime} \subset \mathbf{P}^{d_{1}-g_{X}}$ that determines a smooth point $\left[X^{\prime}\right]$ in the corresponding Hilbert scheme. Now in the open, smooth subset $H$ of this Hilbert scheme consisting of the points which correspond to smooth, irreducible, non-degenerate curves $C \subset \mathbf{P}^{d_{1}-g_{X}}$ of degree $d_{1}=\operatorname{deg} L$ and genus $g_{X}$ with non-special hyperplane section, we have a point $\left[X^{\prime}\right]$ and a non-zero tangent vector corresponding to an embedding $\widetilde{X} \hookrightarrow \mathbf{P}_{\Delta}^{d_{1}-g_{X}}$, extending the embedding $X \hookrightarrow \mathbf{P}^{d_{1}-g_{X}}$, given by the very ample line bundle $\widetilde{L}$. So we can take a smooth irreducible affine curve $T$ in $H$ passing through $\left[X^{\prime}\right]$ and such that its tangent direction is the given tangent vector. Then the pullback to $T$ of the universal family provides a family $(\mathscr{X}, \mathscr{L})$, proper and flat over $T$, whose central fiber is $(X, L)$, whose restriction to the tangent vector to $T$ at 0 is $(\widetilde{X}, \widetilde{L})$ and whose general member $\left(\mathscr{X}_{t}, \mathscr{L}_{t}\right)$ consists of a smooth irreducible projective curve of genus $g_{X}$ and a very ample, non-special line bundle $\mathscr{L}_{t}$ with as many global sections as $L$ and degree $d_{1}=\operatorname{deg} L$.

Step 2. Construction of $\Phi$. We will construct, after shrinking $T$ if necessary, a $T$-morphism

$$
\mathscr{X} \stackrel{\Phi}{\rightarrow} \mathbf{P}_{T}^{r}
$$

whose fiber over the tangent vector to $T$ at 0 is the morphism $\widetilde{X} \stackrel{\widetilde{\varphi}}{\rightarrow} \mathbf{P}_{\Delta}^{r}$ in the statement and whose general fiber $\mathscr{X}_{t} \stackrel{\varphi t}{\longrightarrow} \mathbf{P}^{r}$ for $t \neq 0$ is a closed immersion given by an $r$-dimensional linear subseries of $H^{0}\left(\mathscr{L}_{t}\right)$. 


\section{F. J. Gallego, M. González and B. P. Purnaprajna}

First we look at the properties of $\mathscr{L}$ and of its module of global sections. Let $\mathscr{X} \stackrel{p}{\rightarrow} T$ be the (proper and flat) morphism from $\mathscr{X}$ to $T$. The facts that $p$ is proper, $\mathscr{L}$ is flat over $T$, and $h^{0}\left(\mathscr{L}_{t}\right)=h^{0}(L)$ and $H^{1}\left(\mathscr{X}_{t}, \mathscr{L}_{t}\right)=0$ for every $t \in T$, imply that $p_{*} \mathscr{L}$ is a locally free sheaf of rank $h^{0}(L)$ on $T=\operatorname{Spec} R$ and that 'the formation of $p_{*}$ commutes with base extension'. So we have $\Gamma(\mathscr{L}) \otimes_{R} k[\epsilon] / \epsilon k[\epsilon]=\Gamma(L), \Gamma(\mathscr{L}) \otimes_{R} k[\epsilon] / \epsilon^{2}=\Gamma(\widetilde{L})$ and $\Gamma(\mathscr{L}) \otimes_{R} k(t)=H^{0}\left(\mathscr{X}_{t}, \mathscr{L}_{t}\right)$ for every point $t \in T, t \neq 0$. After shrinking $T$, we can assume that $\Gamma(\mathscr{L})$ is a free $R$-module of rank $h^{0}(L)=n+1$ that induces a $T$-morphism

$$
\mathscr{X} \stackrel{\Psi}{\rightarrow} \mathbf{P}_{T}^{n} .
$$

Let $\widetilde{\Psi}$ be the restriction of $\Psi$ to $\Delta$. Since $\widetilde{\varphi}$ is induced by a set of global sections of $\Gamma(\widetilde{L})$ and $\Gamma(\mathscr{L}) \otimes_{R} k[\epsilon]=\Gamma(\widetilde{L})$, we have that there exists a linear projection

$$
\mathbf{P}_{\Delta}^{n} \stackrel{\widetilde{\rho}}{-\rightarrow} \mathbf{P}_{\Delta}^{r}
$$

such that $\widetilde{\varphi}=\widetilde{\rho} \circ \widetilde{\Psi}$. Now let $t_{1} \in T, t_{1} \neq 0$. Since $\Gamma(\mathscr{L}) \otimes_{R} k\left(t_{1}\right)=H^{0}\left(\mathscr{X}_{t_{1}}, \mathscr{L}_{t_{1}}\right)$, the restriction $\Psi_{t_{1}}$ of $\Psi$ to $t_{1}$ is induced by $H^{0}\left(\mathscr{L}_{t_{1}}\right)$. By step $1, \mathscr{L}_{t_{1}}$ is very ample, so $\Psi_{t_{1}}$ is a closed immersion into $\mathbf{P}_{t_{1}}^{n}$. Since $\mathscr{X}_{t_{1}}$ is a curve and $3 \leqslant r \leqslant h^{0}\left(\mathscr{L}_{t}\right)-1$, it is well known that for a general projection $\rho_{t_{1}}$ the composition $\rho_{t_{1}} \circ \Psi_{t_{1}}$ is again a closed immersion.

Now let $\Sigma=\Delta \cup\left\{t_{1}\right\}$. The pair $\left(\widetilde{\rho}, \rho_{t_{1}}\right)$ is represented by a matrix with coefficients in $\mathscr{O}_{\Sigma}$. Lifting them to $R$ we obtain a linear projection

$$
\mathbf{P}_{T}^{n} \stackrel{\rho}{\stackrel{\rho}{\rightarrow}} \mathbf{P}_{T}^{r}
$$

which restricts to $\widetilde{\rho}$ and to $\rho_{t_{1}}$. We define $\Phi$ as the composition $\rho \circ \Psi$. After maybe shrinking $T$ we may assume that $\Phi$ is a $T$-morphism. After maybe shrinking $T$ again we see that $\Phi$ is a closed immersion when restricted to every $t \in T, t \neq 0$ (see [Gro61, 4.6.7]). By construction the restriction of $\Phi$ to $\Delta$ is $\widetilde{X} \stackrel{\widetilde{\varphi}}{\rightarrow} \mathbf{P}_{\Delta}^{r}$.

Corollary 1.2. Let $X \stackrel{\pi}{\rightarrow} Y$ be a finite cover between a smooth, irreducible curve $X$ and a smooth, irreducible curve $Y$. Then $\pi$ can be realized as the limit of projective embeddings of smooth curves. More precisely, there are embeddings $i$ of $Y$ in projective space so that the composition $\varphi=i \circ \pi$ is the limit of embeddings of smooth curves to projective space.

Proof. Let $\mathscr{E}$ be the trace 0 module of $\pi$. There are always embeddings $i$ of $Y$, induced by complete linear series, into a projective space of sufficiently high dimension so that $H^{1}\left(\mathscr{O}_{Y}(1)\right)=0, H^{1}(\mathscr{E} \otimes$ $\left.\mathscr{O}_{Y}(1)\right)=0$. Then we can apply Theorem 1.1 .

\section{Smoothing of ropes on curves}

In this section we show that ropes of arbitrary multiplicity supported on curves of arbitrary genus can be smoothed, i.e. can be obtained as flat limits of smooth curves, provided they satisfy certain mild conditions which are quite natural from a geometric point of view. We start by recalling the definition of ropes.

Definition 2.1. Let $Y$ be a reduced connected scheme and let $\mathscr{E}$ be a locally free sheaf of rank $m-1$ on $Y$. A rope of multiplicity $m$ (or an $m$-rope, for short), on $Y$ with conormal bundle $\mathscr{E}$ is a scheme $\widetilde{Y}$ with $\tilde{Y}_{\text {red }}=Y$, such that:

(1) $\mathscr{I}_{Y, \widetilde{Y}}^{2}=0$; and

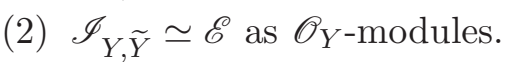

When $\mathscr{E}$ is a line bundle, $\widetilde{Y}$ is called a ribbon on $Y$. 


\section{DEFORMATION OF FINITE MORPHISMS AND SMOOTHING OF ROPES}

By smoothing a rope $\widetilde{Y}$ we mean finding a flat, integral family $\mathscr{Y}$ of schemes over a smooth affine curve $T$, such that, over a point $0 \in T, \mathscr{Y}_{0}=\widetilde{Y}$ and, over the remaining points $t$ of $T, \mathscr{Y}_{t}$ is a smooth, irreducible variety. We will prove that a rope $\widetilde{Y}$ of multiplicity $m$ on $Y$ is smoothable if its conormal bundle $\mathscr{E}$ is the trace 0 module of a smooth, irreducible cover $\pi$ of $Y$ of degree $m$. We will then show that $\widetilde{Y}$ appears as the limit of the images of a family of projective embeddings degenerating to $\pi$. Thus, we will first consider $\widetilde{Y}$ embedded in projective space. Then we use the fact that $\widetilde{Y}$ arises as the central fiber of the image of a first-order infinitesimal deformation of the composition of $\pi$ with the inclusion of $Y$ in projective space $\mathbf{P}^{N}$. Before doing all these, we need to recall the spaces parametrizing ropes and morphisms from ropes of fixed conormal bundle $\mathscr{E}$ to projective space.

TheOREm 2.2. Let $Y$ be a reduced connected scheme and let $\mathscr{E}$ be a vector bundle of rank $m-1$ on $Y$.

(1) A rope $\tilde{Y}$ on $Y$ with conormal bundle $\mathscr{E}$ is determined by an element $\left[e_{\tilde{Y}}\right] \in \operatorname{Ext}_{Y}^{1}\left(\Omega_{Y}, \mathscr{E}\right)$. The rope $\tilde{Y}$ is split (i.e. $Y \hookrightarrow \widetilde{Y}$ admits a retraction) if and only if $\left[e_{\tilde{Y}}\right]=0$.

Assume furthermore that $Y$ is a smooth variety and let $Y \stackrel{i}{\hookrightarrow} Z$ be a closed immersion in another smooth variety $Z$.

(2) There is a one-to-one correspondence between pairs $(\widetilde{Y}, \widetilde{\imath})$, where $\widetilde{Y}$ is a rope on $Y$ with conormal bundle $\mathscr{E}$ and $\widetilde{Y} \stackrel{\widetilde{\imath}}{\rightarrow} Z$ is a morphism extending $Y \stackrel{i}{\hookrightarrow} Z$, and elements $\tau \in \operatorname{Hom}\left(\mathscr{N}_{Y, Z}^{*}, \mathscr{E}\right)$.

(3) If $\tau$ corresponds to a pair $(\widetilde{Y}, \widetilde{\imath}), \widetilde{\imath}$ is an embedding if and only if $\tau$ is a surjective homomorphism.

(4) If $\tau$ corresponds to a pair $(\widetilde{Y}, \widetilde{\imath})$, then $\tau$ is mapped by the connecting homomorphism $\delta$ onto $\left[e_{\widetilde{Y}}\right]$.

Proof. For proofs of the statements see [BE95] and [Gon06, Proposition 2.1].

The construction of the first infinitesimal deformation mentioned above and the relation of ropes and infinitesimal deformations of finite morphisms was done in [Gon06]. We now state a direct consequence of [Gon06, Theorem 3.10].

Theorem 2.3. Let $\widetilde{Y} \subset \mathbf{P}^{r}$ be a rope of multiplicity $m$ on a smooth irreducible curve $Y$ and let $\mathscr{E}$ be the conormal bundle of $\widetilde{Y}$. Assume that there exists a smooth irreducible cover $X \stackrel{\pi}{\rightarrow} Y$ of degree $m$ such that $\pi_{*} \mathscr{O}_{X}=\mathscr{O}_{Y} \oplus \mathscr{E}$. Let $X \stackrel{\varphi}{\rightarrow} \mathbf{P}^{r}$ be the morphism obtained by composing $\pi$ with the inclusion of $Y$ in $\mathbf{P}^{r}$. Then $\widetilde{Y}$ is the central fiber of the image of some first-order infinitesimal deformation of $\varphi$.

Now we use Theorems 1.1 and 2.3 to show that $\widetilde{Y}$ is the limit of the images of a family of embeddings $\Phi_{t}$ of smooth curves, degenerating to $\varphi$. Precisely, we want to extend the infinitesimal deformation of $\varphi$ in such a way that, if we call the image of the family of morphisms $\mathscr{Y} \subset \mathbf{P}^{N} \times T$, then $\mathscr{Y}_{0}=\tilde{Y}$. All this is done in the next theorem.

THEOREM 2.4. Let $\tilde{Y}$ be a rope of multiplicity $m$ and non-negative arithmetic genus, embedded in $\mathbf{P}^{r}(r \geqslant 3)$ as a non-degenerate subscheme, and supported on a smooth irreducible projective curve $Y$. Let $\mathscr{E}$ be the conormal bundle of $\widetilde{Y}$. Assume that $H^{1}\left(\mathscr{O}_{Y}(1)\right)=0$ and $H^{1}\left(\mathscr{E} \otimes \mathscr{O}_{Y}(1)\right)=0$.

If there exists a smooth irreducible cover $X \stackrel{\pi}{\rightarrow} Y$ such that $\pi_{*} \mathscr{O}_{X}=\mathscr{O}_{Y} \oplus \mathscr{E}$, then there exists a family of morphisms $\Phi$ over an affine curve $T$ as described in Theorem 1.1 such that the image $\mathscr{Y}$ of $\Phi$ is a closed integral subscheme $\mathscr{Y} \subset \mathbf{P}_{T}^{r}$, flat over $T$, with the following features:

(1) the general fiber $\mathscr{Y}_{t}, t \in T-0$, is a smooth irreducible projective non-degenerate curve with non-special hyperplane section in $\mathbf{P}^{r}$;

(2) the central fiber $\mathscr{Y}_{0} \subset \mathbf{P}^{r}$ is $\tilde{Y} \subset \mathbf{P}^{r}$. 


\section{F. J. Gallego, M. González and B. P. Purnaprajna}

Remark 2.5. The hypothesis in Theorem 2.4 that $\tilde{Y}$ be embedded in $\mathbf{P}^{r}$ as non-degenerate subscheme can be relaxed. Indeed, one only needs to ask that $h^{0}\left(\pi^{*} \mathscr{O}_{Y}(1)\right) \geqslant r+1$ (see (2.5.1) below).

Proof of Theorem 2.4. We use the notation of the proof of Theorem 1.1. For the line bundle $L=$ $\pi^{*} \mathscr{O}_{Y}(1)$ on $X$ we have

$$
H^{0}(L)=H^{0}\left(\mathscr{O}_{Y}(1)\right) \oplus H^{0}\left(\mathscr{E} \otimes \mathscr{O}_{Y}(1)\right)
$$

Moreover, from the sequence

$$
0 \longrightarrow \mathscr{E}^{\longrightarrow} \mathscr{O}_{\widetilde{Y}} \longrightarrow \mathscr{O}_{Y} \longrightarrow 0
$$

twisted by $\mathscr{O}_{\widetilde{Y}}(1)$ and the assumption $H^{1}\left(\mathscr{E} \otimes \mathscr{O}_{Y}(1)\right)=0$ we obtain an isomorphism

$$
H^{0}\left(\mathscr{O}_{\widetilde{Y}}(1)\right)=H^{0}\left(\mathscr{O}_{Y}(1)\right) \oplus H^{0}\left(\mathscr{E} \otimes \mathscr{O}_{Y}(1)\right) .
$$

By assumption $\tilde{Y}$ is embedded in $\mathbf{P}^{r}$ as a non-degenerate subscheme, so $H^{0}\left(\mathscr{O}_{\mathbf{P}^{r}}(1)\right) \subset H^{0}\left(\mathscr{O}_{\widetilde{Y}}(1)\right)$. Thus we obtain the inequality

$$
h^{0}(L) \geqslant r+1
$$

Moreover from Theorem 2.3 we know that there exists a first-order infinitesimal deformation

$$
\widetilde{X} \stackrel{\widetilde{\varphi}}{\rightarrow} \mathbf{P}_{\Delta}^{r}
$$

of $\varphi$ such that the central fiber of the image of $\widetilde{\varphi}$ is equal to the rope $\tilde{Y}$.

Therefore there exist a family $\mathscr{X} \rightarrow T$ and a $T$-morphism $\mathscr{X} \stackrel{\Phi}{\rightarrow} \mathbf{P}_{T}^{r}$ as in Theorem 1.1. Let $\mathscr{Y}$ be the image of the $T$-morphism $\mathscr{X} \stackrel{\Phi}{\rightarrow} \mathbf{P}_{T}^{r}$. The total family $\mathscr{X}$ is smooth and irreducible so $\mathscr{Y}$ is integral. Furthermore, $\Phi$ is a closed immersion over $T-0$ since, by Theorem 1.1, $\Phi_{t}$ is a closed immersion for every $t \in T-0$ (see e.g. [Gro61, 4.6.7]). Therefore for $t \in T-0$ we have the equality $\mathscr{Y}_{t}=\operatorname{im}\left(\Phi_{t}\right)$. Since $\mathscr{X}_{t}$ is smooth, this proves feature (1). Finally, the fact that $T$ is an integral smooth curve and $\mathscr{Y}$ is integral and dominates $T$ imply that $\mathscr{Y}$ is flat over $T$. So the fiber $\mathscr{Y} 0$ of $\mathscr{Y}$ at $0 \in T$ is the flat limit of the images of $\mathscr{X}_{t} \stackrel{\Phi_{t}}{\longrightarrow} \mathbf{P}^{r}$ for $t \neq 0$. Moreover, this fiber $\mathscr{Y}_{0}$ contains the central fiber $(\operatorname{im} \widetilde{\varphi})_{0}$ of the image of $\widetilde{\varphi}$. Since $\widetilde{Y}$ has conormal bundle $\mathscr{E}$ and $\pi$ has trace 0 module $\mathscr{E}$, the genus of $X$ and the arithmetic genus of $\widetilde{Y}$ are equal. Then $\mathscr{Y}_{0}$ and $(\operatorname{im} \widetilde{\varphi})_{0}$ have the same degree and the same arithmetic genus, so they are equal.

Remark 2.6. In the vast majority of cases, for a fixed inclusion $\widetilde{Y} \stackrel{\tilde{\imath}}{\hookrightarrow} \mathbf{P}^{r}$, there are many different smoothings of $\widetilde{Y}$, as follows.

(1) There will be many possible covers $\pi$ to choose.

(2) Once $\pi$ is chosen and $\varphi$ is therefore fixed, the element $\tau \in \operatorname{Hom}\left(\mathscr{N}_{Y, Z}^{*}, \mathscr{E}\right)$ corresponding to $(\tilde{Y}, \tilde{\imath})$ may have, in most cases, many different liftings to $H^{0}\left(\mathscr{N}_{\varphi}\right)$. Liftings $\mu$ of $\tau$ to $H^{0}\left(\mathscr{N}_{\varphi}\right)$ correspond to first-order infinitesimal deformations $\widetilde{\varphi}$ of $\varphi$, such that $(\operatorname{im} \widetilde{\varphi})_{0}=\widetilde{Y}$. For details on this, see [Gon06, §3].

(3) There are many different ways of extending $\widetilde{\varphi}$ to a family of morphisms $\Phi$, as the proof of Theorem 1.1 shows.

COROLlary 2.7. Let $\widetilde{Y}$ be a rope of multiplicity $m$ on a smooth irreducible projective curve $Y$, with conormal bundle $\mathscr{E}$ and non-negative arithmetic genus. If there is a smooth connected cover $X \stackrel{\pi}{\rightarrow} Y$ such that $\pi_{*} \mathscr{O}_{X}=\mathscr{O}_{Y} \oplus \mathscr{E}$, then $\widetilde{Y}$ is smoothable.

Proof. Since $\widetilde{Y}$ is a proper scheme of dimension 1, it is projective. Taking $r$ sufficiently large we can embed $\widetilde{Y}$ as a non-degenerate subscheme of $\mathbf{P}^{r}$ and, at the same time, make $H^{1}\left(\mathscr{O}_{Y}(1)\right)=$ $H^{1}\left(\mathscr{E} \otimes \mathscr{O}_{Y}(1)\right)=0$. Then the corollary follows from Theorem 2.4. 


\section{DEFORMATION OF FINITE MORPHISMS AND SMOOTHING OF ROPES}

\section{Embeddings of multiplicity 3 ropes in projective space}

Proper schemes of dimension 1 are projective, so in particular, any multiplicity 3 rope $\tilde{Y}$ on $\mathbf{P}^{1}$ can be embedded in a sufficiently large projective space $\mathbf{P}^{N}$. The main purpose of this section is to find, given $\widetilde{Y}$, the smallest $N$ so that $\widetilde{Y}$ can be embedded in $\mathbf{P}^{N}$.

The conormal bundle of a rope $\widetilde{Y}$ of multiplicity 3 is a vector bundle $\mathscr{E}$ of rank 2 . The bundle $\mathscr{E}$ splits as a direct sum $\mathscr{O}_{\mathbf{P}^{1}}(-a) \oplus \mathscr{O}_{\mathbf{P}^{1}}(-b)$, where $a$ and $b$ are integers and $a \geqslant b$. The arithmetic genus of $\widetilde{Y}$ is $a+b-2$. Thus, if we set an arithmetic genus $g$, there are ropes of genus $g$ with different conormal bundles. We can set a hierarchy among them, as follows.

Definition 3.1. Let $\widetilde{Y}$ be a rope on $\mathbf{P}^{1}$ with conormal bundle $\mathscr{E}=\mathscr{O}_{\mathbf{P}^{1}}(-a) \oplus \mathscr{O}_{\mathbf{P}^{1}}(-b), a \geqslant b$.

(1) By analogy with smooth trigonal curves, we say that the Maroni invariant of $\tilde{Y}$ is $n=a-b$.

(2) We say that $\widetilde{Y}$ is balanced if $n=0$ or $n=1$. Let the ropes $\widetilde{Y}_{1}$ and $\widetilde{Y}_{2}$ have the same arithmetic genus and let $n_{i}$ be the Maroni invariant of $\widetilde{Y}_{i}$. We say that $\widetilde{Y}_{1}$ is more balanced than $\widetilde{Y}_{2}$ if $n_{1} \leqslant n_{2}$.

In $\S 4$ (see Proposition 4.4), we will show that a less balanced rope is a degeneration of more balanced ropes.

Now we proceed to describe the embeddings of ropes of multiplicity 3 on $\mathbf{P}^{1}$. We start looking for embeddings supported on a rational normal curve lying in the same projective space where the rope is embedded. Obviously, a rope of multiplicity 3 cannot be embedded in $\mathbf{P}^{2}$. There is not much room in $\mathbf{P}^{3}$ either. Indeed, the only 3-ropes in $\mathbf{P}^{3}$, supported on the twisted cubic are the non-split ropes on $\mathbf{P}^{1}$ with conormal bundle $\mathscr{O}_{\mathbf{P}^{1}}(-5) \oplus \mathscr{O}_{\mathbf{P}^{1}}(-5)$. This is because the conormal bundle of the twisted cubic is $\mathscr{O}_{\mathbf{P}^{1}}(-5) \oplus \mathscr{O}_{\mathbf{P}^{1}}(-5)$ and the map $\delta$ in (3.4.1) below is, in this case, an isomorphism. Thus we start looking for embeddings in projective spaces of dimension 4 or higher. More precisely, we will find the smallest $N$ so that all ropes with fixed conormal bundle $\mathscr{E}$ can be embedded in $\mathbf{P}^{N}$.

Theorem 3.2. Let $\widetilde{Y}$ be a rope on $Y=\mathbf{P}^{1}$ of arithmetic genus $g$, Maroni invariant $n$ and conormal bundle $\mathscr{E}=\mathscr{O}_{\mathbf{P}^{1}}(-a) \oplus \mathscr{O}_{\mathbf{P}^{1}}(-b), a \geqslant b$. Let $N_{0}=\max \{4, a-1\}$ (i.e. $\left.N_{0}=\max \{4,(g+n) / 2\}\right)$. Let $Y \stackrel{i}{\hookrightarrow} \mathbf{P}^{N}$ be an embedding of $Y$ as a rational normal curve in $\mathbf{P}^{N}$. If $N \geqslant N_{0}$, then there are embeddings $\tilde{Y} \stackrel{\tilde{\imath}}{\hookrightarrow} \mathbf{P}^{N}$ that extend $i$. In particular, $\widetilde{Y}$ can be embedded as a subscheme of $\mathbf{P}^{N}$, supported on a rational normal curve of degree $N$.

Remark 3.3. Under the hypotheses of Theorem 3.2:

(1) $H^{1}\left(\mathscr{E} \otimes \mathscr{O}_{\mathbf{P}^{1}}(N)\right)=0$;

(2) $H^{0}\left(\mathscr{E} \otimes \mathscr{O}_{\mathbf{P}^{1}}(N)\right)=0$ if and only if $\mathscr{E}=\mathscr{O}_{\mathbf{P}^{1}}(-a) \oplus \mathscr{O}_{\mathbf{P}^{1}}(-a)$ (i.e. if $g$ is even and $n=0$ ) and $N=a-1$ (i.e. $N=g / 2$ ).

In particular, $\tilde{\imath}$ embeds $\tilde{Y}$ as a linearly normal subscheme of $\mathbf{P}^{N}$ if and only if $\mathscr{E}=\mathscr{O}_{\mathbf{P}^{1}}(-a) \oplus$ $\mathscr{O}_{\mathbf{P}^{1}}(-a)$ and $N=a-1$.

In order to prove Theorem 3.2 we need the following result.

Lemma 3.4. Let $Y$ be an irreducible projective variety and let $\mathscr{E}$ and $\mathscr{F}$ be locally free sheaves of finite rank on $Y$.

(1) The surjective homomorphisms from $\mathscr{F}$ to $\mathscr{E}$ form an open set of $\operatorname{Hom}(\mathscr{F}, \mathscr{E})$ which is the complement of an algebraic cone.

(2) Consider an extension of a coherent sheaf $\mathscr{F}^{\prime}$ by $\mathscr{F}$. Let $\operatorname{Hom}(\mathscr{F}, \mathscr{E}) \stackrel{\delta}{\rightarrow} \operatorname{Ext}^{1}\left(\mathscr{F}^{\prime}, \mathscr{E}\right)$ be the connecting map induced by the extension. If the class of the split extension lifts to an epimorphism, then every class in the image of $\delta$ can be lifted to an epimorphism. 


\section{F. J. Gallego, M. González and B. P. Purnaprajna}

In particular, if $Y \stackrel{i}{\hookrightarrow} Z$ is an inclusion of smooth projective varieties, and the split rope on $Y$ with conormal bundle $\mathscr{E}$ admits an embedding into $Z$ extending $i$, then every rope on $Y$ with conormal bundle $\mathscr{E}$ which admits a morphism to $Z$ extending $i$ also admits an embedding into $Z$ extending $i$.

Proof. The lemma is a generalization of [Gon06, Lemma 4.1] and its proof follows almost word for word the proof given there. The conclusion about the embeddings of ropes follows from part (2) and Theorem 2.2.

Proof of Theorem 3.2. Recall the sequence

$$
\operatorname{Hom}\left(\left.\Omega_{\mathbf{P}^{N}}\right|_{\mathbf{P}^{1}}, \mathscr{E}\right) \stackrel{\gamma}{\rightarrow} \operatorname{Hom}\left(\mathscr{I} / \mathscr{I}^{2}, \mathscr{E}\right) \stackrel{\delta}{\rightarrow} \operatorname{Ext}^{1}\left(\Omega_{Y}, \mathscr{E}\right) \longrightarrow \operatorname{Ext}^{1}\left(\left.\Omega_{\mathbf{P}^{N}}\right|_{\mathbf{P}^{1}}, \mathscr{E}\right) .
$$

The bundle $\left.\Omega_{\mathbf{P}^{N}}\right|_{\mathbf{P}^{1}}=\mathscr{O}_{\mathbf{P}^{1}}(-N-1)^{\oplus N}$, hence we have

$$
\operatorname{Ext}^{1}\left(\left.\Omega_{\mathbf{P}^{N}}\right|_{\mathbf{P}^{1}}, \mathscr{E}\right)=H^{1}\left(\mathscr{O}_{\mathbf{P}^{1}}(N+1-a)^{\oplus N} \oplus \mathscr{O}_{\mathbf{P}^{1}}(N+1-b)^{\oplus N}\right)=0 .
$$

This means that any $\widetilde{Y}$ with conormal bundle $\mathscr{E}$ admits a morphism to $\mathbf{P}^{N}$ extending $i$. We are going to prove that, among these morphisms, one can actually find embeddings. By Lemma 3.4, it is enough to show that the split rope with conormal $\mathscr{E}$ possesses such an embedding, i.e. it is enough to show that there exists an epimorphism $\tau \in \operatorname{Hom}\left(\mathscr{I} / \mathscr{I}^{2}, \mathscr{E}\right)$ such that $\delta(\tau)=0$. This is the same as finding an epimorphism $\tau$ in the image of $\gamma$.

Let $\mathscr{I}$ denote the ideal sheaf of the image of $Y$ in $\mathbf{P}^{N}$. Then the conormal bundle

$$
\mathscr{I} / \mathscr{I}^{2}=\mathscr{O}_{\mathbf{P}^{1}}(-N-2)^{\oplus N-1} \text {. }
$$

Moreover $\mathscr{I} / \mathscr{I}^{2}$ fits in the following exact sequence:

$$
0 \longrightarrow \mathscr{I} /\left.\mathscr{I}^{2} \longrightarrow \Omega_{\mathbf{P}^{N}}\right|_{Y} \longrightarrow \Omega_{Y} \longrightarrow 0,
$$

which is the same as

$$
0 \longrightarrow \mathscr{O}_{\mathbf{P}^{1}}(-N-2)^{\oplus N-1} \stackrel{\alpha}{\rightarrow} \mathscr{O}_{\mathbf{P}^{1}}(-N-1)^{\oplus N} \stackrel{\beta}{\rightarrow} \mathscr{O}_{\mathbf{P}^{1}}(-2) \longrightarrow 0 .
$$

If we fix a suitable 'basis' for each bundle in (3.4.2), the matrix of $\beta$ is

$$
\left(\begin{array}{lllll}
X_{0}^{N-1} & X_{0}^{N-2} X_{1} & \ldots & X_{0} X_{1}^{N-2} & X_{1}^{N-1}
\end{array}\right)
$$

and the matrix of $\alpha$ is the $N \times(N-1)$ matrix

$$
\left(\begin{array}{ccccccc}
X_{1} & 0 & 0 & 0 & \ldots & 0 & 0 \\
-X_{0} & X_{1} & 0 & 0 & \ldots & 0 & 0 \\
0 & -X_{0} & X_{1} & 0 & \ldots & 0 & 0 \\
\vdots & \vdots & \vdots & \vdots & \ddots & \vdots & \vdots \\
0 & 0 & 0 & 0 & \ldots & -X_{0} & X_{1} \\
0 & 0 & 0 & 0 & \ldots & 0 & -X_{0}
\end{array}\right)
$$

where $X_{0}, X_{1}$ are homogeneous coordinates of $\mathbf{P}^{1}$. On the other hand, an arbitrary element of $\operatorname{Hom}\left(\left.\Omega_{\mathbf{P}^{N}}\right|_{Y}, \mathscr{E}\right)$ has a matrix of the form

$$
\left(\begin{array}{llll}
a_{1} & a_{2} & \ldots & a_{N} \\
b_{1} & b_{2} & \ldots & b_{N}
\end{array}\right)
$$

where the $a_{i}$ and $b_{j}$ are homogeneous forms on $\mathbf{P}^{1}$. The forms $a_{1}, \ldots, a_{N}$ have degree $N-a+1$ (which is a non-negative integer) and $b_{1}, \ldots, b_{N}$ have degree $N-b+1$.

Then the image of $\gamma$, which is the kernel of $\delta$, consists of homomorphisms in $\operatorname{Hom}\left(\mathscr{I} / \mathscr{I}^{2}, \mathscr{E}\right)$ whose matrices have size $2 \times(N-1)$ and are of the form

$$
M=\left(\begin{array}{cccc}
a_{1} X_{1}-a_{2} X_{0} & a_{2} X_{1}-a_{3} X_{0} & \ldots & a_{N-1} X_{1}-a_{N} X_{0} \\
b_{1} X_{1}-b_{2} X_{0} & b_{2} X_{1}-b_{3} X_{0} & \ldots & b_{N-1} X_{1}-b_{N} X_{0}
\end{array}\right),
$$




\section{DEFORMATION OF FINITE MORPHISMS AND SMOOTHING OF ROPES}

where $a_{1}, \ldots, a_{N}, b_{1}, \ldots, b_{N}$ are as above. Then, as far as $N \geqslant 4$, we can always find $a_{i}$ and $b_{j}$ so that $M$ has maximal rank 2 at every point of $\mathbf{P}^{1}$ and corresponds therefore to an element of $\operatorname{Hom}\left(\mathscr{I} / \mathscr{I}^{2}, \mathscr{E}\right)$ which is an epimorphism and whose image by $\delta$ is 0 .

In the next theorem we show that the bound on $N$ obtained in Theorem 3.2 is the best possible that allows all ropes $\widetilde{Y}$ with a fixed conormal bundle $\mathscr{E}$ to be embedded in $\mathbf{P}^{N}$. The theorem also characterizes, among other things, what ropes can be embedded in smaller projective spaces.

TheOREM 3.5. Let $\tilde{Y}$ be a rope on $Y=\mathbf{P}^{1}$ with conormal bundle $\mathscr{E}=\mathscr{O}_{\mathbf{P}^{1}}(-a) \oplus \mathscr{O}_{\mathbf{P}^{1}}(-b), a \geqslant b$. Let $Y \stackrel{i}{\hookrightarrow} \mathbf{P}^{N}$ be an embedding of $Y$ as a rational normal curve in $\mathbf{P}^{N}$.

(1) If $N=a-2$ and $\tilde{Y}$ does not admit a projection onto a ribbon of conormal bundle $\mathscr{O}_{\mathbf{P}^{1}}(-b)$, then there exist embeddings $\tilde{Y} \stackrel{\tilde{\imath}}{\hookrightarrow} \mathbf{P}^{N}$ which extend $i$.

(2) If $N=a-2$ and $\widetilde{Y}$ admits a projection onto a ribbon $\widehat{Y}$ of conormal $\mathscr{O}_{\mathbf{P}^{1}}(-b)$, then there exist morphisms $\tilde{Y} \stackrel{\tilde{s}}{\rightarrow} \mathbf{P}^{N}$ extending $i$ and all of them factor through a projection onto a ribbon whose conormal bundle is a subsheaf of $\mathscr{O}_{\mathbf{P}^{1}}(-b)$.

(3) If $N \leqslant a-3$, then there exist morphisms $\tilde{Y} \stackrel{\tilde{\imath}}{\rightarrow} \mathbf{P}^{N}$ extending $i$ only if $\tilde{Y}$ admits a projection $p$ onto a ribbon $\widehat{Y}$ whose conormal bundle is a subsheaf of $\mathscr{O}_{\mathbf{P}^{1}}(-b)$. These morphisms factor through $\widehat{Y}$.

In particular, if $N<a-1$, the rope $\tilde{Y}$ can be embedded in $\mathbf{P}^{N}$ supported on a rational normal curve of degree $N$ if and only if $N=a-2$ and $\widetilde{Y}$ does not admit a projection onto a ribbon with conormal bundle $\mathscr{O}_{\mathbf{P}^{1}}(-b)$.

Proof. Let $\mathscr{I}$ be the ideal sheaf of $i(Y)$ in $\mathbf{P}^{N}$. Recall that the conormal bundle of $i(Y)$ in $\mathbf{P}^{N}$ is $\mathscr{I} / \mathscr{I}^{2}=\mathscr{O}_{\mathbf{P}^{1}}(-N-2)^{\oplus N-1}$. We see first under what conditions $i$ can be extended to a morphism $\tilde{Y} \stackrel{\tilde{\imath}}{\rightarrow} \mathbf{P}^{N}$, for some rope $\tilde{Y}$ with conormal bundle $\mathscr{E}$. Recall that, by Theorem 2.2 , the space $\operatorname{Hom}\left(\mathscr{I} / \mathscr{I}^{2}, \mathscr{E}\right)$ parametrizes pairs $(\widetilde{Y}, \tilde{\imath})$, where $\tilde{\imath}$ extends $i$.

If $N \leqslant b-3$, then $\operatorname{Hom}\left(\mathscr{I} / \mathscr{I}^{2}, \mathscr{E}\right)=0$, so $i$ can be extended if and only if $\tilde{Y}$ is the split rope. In this case it is clear that $\tilde{\imath}$ factors through the retraction of the split rope onto $Y$. If $b-2 \leqslant N \leqslant a-3$, any homomorphism from $\mathscr{I} / \mathscr{I}^{2}$ to $\mathscr{E}$ is the composition of a homomorphism from $\mathscr{I} / \mathscr{I}^{2}$ to $\mathscr{O}_{\mathbf{P}^{1}}(-b)$, followed by the bundle embedding $\mathscr{O}_{\mathbf{P}^{1}}(-b) \hookrightarrow \mathscr{E}$. This means that any extension of $i$ to a rope $\widetilde{Y}$ with conormal bundle $\mathscr{E}$ factors through an extension (actually, an embedding, provided the corresponding homomorphism from $\mathscr{I} / \mathscr{I}^{2}$ to $\mathscr{E}$ is non-zero) of $i$ to a ribbon $\widehat{Y}$, whose conormal bundle is a subsheaf of $\mathscr{O}_{\mathbf{P}^{1}}(-b)$. In particular, $\widetilde{Y}$ admits a projection onto $\widehat{Y}$. This proves part (3).

We now prove part (1). If $N=a-2$, then $\operatorname{Ext}^{1}\left(\left.\Omega_{\mathbf{P}^{N}}\right|_{\mathbf{P}^{1}}, \mathscr{E}\right)=0$. Therefore, given any rope $\tilde{Y}$ with conormal bundle $\mathscr{E}$, the morphism $i$ can be extended to $\tilde{Y}$. Let us now fix $\widetilde{Y}$, which corresponds to some $\zeta \in \operatorname{Ext}^{1}\left(\Omega_{\mathbf{P}^{1}}, \mathscr{E}\right)$, and look at the possible extensions of $i$ to $\widetilde{Y}$, which correspond to elements $\tau \in \operatorname{Hom}\left(\mathscr{I} / \mathscr{I}^{2}, \mathscr{E}\right)$ mapping to $\zeta$. As argued in the proof of Theorem 3.2, $\tau$ can be thought of as a $2 \times(N-1)$ matrix, in which the entries of the first row are constant and the entries of the second row are homogeneous forms of degree $a-b$. If all the entries of the first row are zeros, then $\tau$ corresponds to an extension $\tilde{Y} \stackrel{\tilde{\imath}}{\rightarrow} \mathbf{P}^{N}$ that factors through a ribbon whose conormal bundle is a subsheaf of $\mathscr{O}_{\mathbf{P}^{1}}(-b)$. On the other hand, an element in $\operatorname{Hom}\left(\left.\Omega_{\mathbf{P}^{N}}\right|_{\mathbf{P}^{1}}, \mathscr{E}\right)$ corresponds to a $2 \times N$ matrix with zero entries in its first row and homogeneous forms of degree $a-b-1$ in its second row. Arguing as in the proof of Theorem 3.2, we can then add to $\tau$ a suitable element of the kernel of $\delta$, and obtain an element $\tau^{\prime}$ in $\operatorname{Hom}\left(\mathscr{I} / \mathscr{I}^{2}, \mathscr{E}\right)$, such that $\delta\left(\tau^{\prime}\right)=\zeta$, and whose image is $\mathscr{O}_{\mathbf{P}^{1}}(-b)$. In this situation, $\tau^{\prime}$ corresponds to an extension of $i$ to $\tilde{Y}$, which factors through an embedding of a ribbon $\widehat{Y}$ with conormal bundle $\mathscr{O}_{\mathbf{P}^{1}}(-b)$. In particular, $\widetilde{Y}$ would admit a projection onto $\widehat{Y}$. 


\section{F. J. Gallego, M. González and B. P. Purnaprajna}

Now, to prove part (1), assume that $\tilde{Y}$ does not admit a projection onto such $\widehat{Y}$. Then any preimage $\tau$ of $\zeta$ by $\delta$ must correspond to a matrix whose first row is non-zero. Adding as before a suitable element from the kernel of $\delta$ we can actually assume that $\tau$ is a surjective homomorphism of $\operatorname{Hom}\left(\mathscr{I} / \mathscr{I}^{2}, \mathscr{E}\right)$, so $i$ can be lifted to an embedding of $\widetilde{Y}$ into $\mathbf{P}^{N}$.

Finally, to prove part (2), assume that $\widetilde{Y}$ admits a projection $p$ onto a ribbon $\widehat{Y}$ with conormal bundle $\mathscr{O}_{\mathbf{P}^{1}}(-b)$. As before $\widetilde{Y}$ corresponds to an element $\zeta \in \operatorname{Ext}^{1}\left(\Omega_{\mathbf{P}^{1}}, \mathscr{E}\right)$. Since $\operatorname{Ext}^{1}\left(\left.\Omega_{\mathbf{P}^{N}}\right|_{\mathbf{P}^{1}}\right.$, $\left.\mathscr{O}_{\mathbf{P}^{1}}(-b)\right)=0, i$ can be extended to a morphism $\hat{i}$ from $\hat{Y}$. Composing $\hat{i}$ with $p$, we get also an extension of $i$ to $\tilde{Y}$. This extension corresponds to an element $\tau \in \operatorname{Hom}\left(\mathscr{I} / \mathscr{I}^{2}, \mathscr{E}\right)$ whose corresponding matrix has zero entries in its first row. Now any other $\tau^{\prime}$ mapping to $\zeta$ will differ from $\tau$ by an element in the kernel of $\delta$. Arguing as in the previous paragraph we see that the matrix of such $\tau^{\prime}$ will also have zeros in its first row. This means that all extensions of $i$ will factor through ribbons whose conormal is a subsheaf of $\mathscr{O}_{\mathbf{P}^{1}}(-b)$. This completes the proof of part $(2)$.

As noted in Remark 3.3, the embeddings in Theorem 3.2 are almost never linearly normal. However, as a corollary of Theorem 3.2, we obtain an effective bound for linearly normal embeddings of ropes in projective space.

Corollary 3.6. Let $\tilde{Y}$ be a rope on $Y=\mathbf{P}^{1}$ with arithmetic genus $g$, Maroni invariant $n$ and conormal bundle $\mathscr{E}=\mathscr{O}_{\mathbf{P}^{1}}(-a) \oplus \mathscr{O}_{\mathbf{P}^{1}}(-b)$, $a \geqslant b$. Let $N_{0}=\max \{4, a-1\}$ (i.e. $N_{0}=$ $\max \{4,(g+n) / 2\})$. Let $Y \stackrel{i}{\hookrightarrow} \mathbf{P}^{N} \subset \mathbf{P}^{M}$ be an embedding of $Y$ as a rational normal curve in $\mathbf{P}^{N}$, where $M=N+h^{0}\left(\mathscr{E} \otimes \mathscr{O}_{\mathbf{P}^{1}}(N)\right)$. If $N \geqslant N_{0}$, then there are embeddings $\tilde{Y} \stackrel{\tilde{J}}{\hookrightarrow} \mathbf{P}^{M}$ extending $i$ such that $\tilde{\jmath}$ embeds $\widetilde{Y}$ as a linearly normal subscheme of $\mathbf{P}^{M}$.

Remark 3.7. Under the hypotheses of Corollary 3.6 we have $H^{1}\left(\mathscr{E} \otimes \mathscr{O}_{\mathbf{P}^{1}}(N)\right)=0$.

Proof of Corollary 3.6. Consider one of the embeddings $\tilde{Y} \stackrel{\tilde{\imath}}{\hookrightarrow} \mathbf{P}^{N}$ obtained in Theorem 3.2. Consider also the sequence

$$
0 \longrightarrow H^{0}\left(\mathscr{E} \otimes \mathscr{O}_{\mathbf{P}^{1}}(N)\right) \longrightarrow H^{0}\left(\tilde{\imath}^{*} \mathscr{O}_{\mathbf{P}^{N}}(1)\right) \longrightarrow H^{0}\left(\mathscr{O}_{\mathbf{P}^{1}}(N)\right) \longrightarrow 0
$$

which is right exact by Remark 3.7. Then the complete linear series of $\tilde{\imath}^{*} \mathscr{O}_{\mathbf{P}^{N}}(1)$ induces a morphism $\widetilde{Y} \stackrel{\tilde{J}}{\rightarrow} \mathbf{P}^{M}$, which is an embedding because $\widetilde{\imath}$ also is.

\section{Smoothing of multiplicity 3 ropes on $\mathbf{P}^{1}$}

In this section we apply the results of $\S \S 2$ and 3 to prove, in a very precise way, that ropes on $\mathbf{P}^{1}$ of multiplicity 3 and non-negative arithmetic genus are smoothable. Let $\widetilde{Y}$ be a rope of multiplicity 3 on $\mathbf{P}^{1}$ and let $\mathscr{E}=\mathscr{O}_{\mathbf{P}^{1}}(-a) \oplus \mathscr{O}_{\mathbf{P}^{1}}(-b)$ be its conormal bundle. If we want to apply Theorem 2.4 to $\widetilde{Y}$, we need a smooth, irreducible triple cover $X \stackrel{\pi}{\rightarrow} \mathbf{P}^{1}$, with trace 0 module $\mathscr{E}$. This imposes conditions on $a$ and $b$, which we summarize in the following well-known result (see [Mir85]).

Theorem 4.1. Let $\mathscr{E}=\mathscr{O}_{\mathbf{P}^{1}}(-a) \oplus \mathscr{O}_{\mathbf{P}^{1}}(-b)$ be a vector bundle of rank 2 on $\mathbf{P}^{1}$. There exists a smooth, irreducible cover $X \stackrel{\pi}{\rightarrow} \mathbf{P}^{1}$ with $\pi_{*} \mathscr{O}_{X}=\mathscr{O}_{\mathbf{P}^{1}} \oplus \mathscr{E}$ if and only if $a>0, b>0, a \leqslant 2 b$ and $b \leqslant 2 a$. In this case, the general triple cover with trace 0 module $\mathscr{E}$ is smooth and irreducible.

Theorems 2.4 and 4.1 allow us to say at once that ropes of genus $g \geqslant 0$, embedded in projective space, with a conormal bundle $\mathscr{E}$ satisfying the above conditions, can be smoothed. These are ropes whose Maroni invariant $n$ is small compared to the genus (precisely, $n \leqslant(g+2) / 3$ ). However, this restriction can be circumvented by degenerating more balanced ropes (to which a smooth triple cover can be associated) to less balanced ropes (to which a smooth triple cover cannot be associated). This is done in the following definition and propositions. 


\section{DEFORMATION OF FINITE MORPHISMS AND SMOOTHING OF ROPES}

Definition 4.2. Let $\mathscr{Y}$ and $U$ be reduced and connected $\mathbf{k}$-schemes, let $\mathscr{Y} \stackrel{p}{\rightarrow} U$ be a smooth proper morphism with connected fibers and let $\mathbb{E}$ be a locally free sheaf on $\mathscr{Y}$. A rope on $\mathscr{Y}$ relative over $U$, with conormal bundle $\mathbb{E}$, is a scheme over $U, \widetilde{\mathscr{Y}} \stackrel{\tilde{p}}{\rightarrow} U$, where $\widetilde{\mathscr{Y}}$ is a rope on $\mathscr{Y}$ with conormal bundle $\mathbb{E}$ and $p$ is the composition of the closed immersion $\mathscr{Y} \hookrightarrow \widetilde{\mathscr{Y}}$ followed by $\tilde{p}$.

Proposition 4.3. Let $\mathscr{Y} \stackrel{p}{\rightarrow} U, \widetilde{\mathscr{Y}} \stackrel{\tilde{p}}{\rightarrow} U$ and $\mathbb{E}$ be as in Definition 4.2.

(1) The ropes on $\mathscr{Y}$ relative over $U$ with conormal bundle $\mathbb{E}$, up to equivalence of extensions, are classified by the space $\operatorname{Ext}_{\mathscr{Y}}^{1}\left(\Omega_{\mathscr{Y} / U}, \mathbb{E}\right)$.

(2) If $U$ is affine, then there is a natural isomorphism between $\operatorname{Ext}_{\mathscr{Y}}^{1}\left(\Omega_{\mathscr{Y} / U}, \mathbb{E}\right)$ and $H^{0}\left(R^{1} p_{*}\right.$ $\left.\left(\Omega_{\mathscr{Y} / U}^{*} \otimes \mathbb{E}\right)\right)$.

(3) The morphism $\widetilde{\mathscr{Y}} \stackrel{\tilde{p}}{\rightarrow} U$ is a proper and flat family of ropes $\widetilde{\mathscr{Y}}_{u}$ on $\mathscr{Y}_{u}$ with conormal bundle $\mathbb{E}_{u}=\mathbb{E} \otimes \mathscr{O}_{\mathscr{Y}_{u}}, u \in U$

Let $\mathscr{Z}$ be a smooth, proper scheme over $U$, let $\mathscr{Y} \stackrel{i}{\hookrightarrow} \mathscr{Z}$ be a $U$-morphism which is a closed immersion of smooth varieties and let $\mathscr{I}$ be the ideal sheaf of $i(\mathscr{Y})$ in $\mathscr{Z}$.

(4) The $U$-morphisms $\tilde{\imath}$ extending $i$ to $\widetilde{\mathscr{Y}}$ are classified by $\operatorname{Hom}_{\mathscr{Y}}\left(\mathscr{I} / \mathscr{I}^{2}, \mathbb{E}\right)$.

(5) If $(\widetilde{\mathscr{Y}}, \tilde{\imath})$ corresponds to $\tau \in \operatorname{Hom}_{\mathscr{Y}}\left(\mathscr{I} / \mathscr{I}^{2}, \mathbb{E}\right)$, then $\tilde{\imath}$ is a closed immersion if and only if $\tau$ is a surjective homomorphism. In this case, $\tilde{\imath}$ is a family of closed immersions of ropes.

(6) If $(\widetilde{\mathscr{Y}}, \tilde{\imath})$ corresponds to $\tau \in \operatorname{Hom}_{\mathscr{Y}}\left(\mathscr{I} / \mathscr{I}^{2}, \mathbb{E}\right)$ and $\widetilde{\mathscr{Y}}$ corresponds to $\varepsilon \in \operatorname{Ext}_{\mathscr{Y}}^{1}\left(\Omega_{\mathscr{Y}} / U, \mathbb{E}\right)$, then $\varepsilon$ is the image of $\tau$ by the connecting homomorphism.

Proof. Part (1) is a relative version of Theorem 2.2(1) and the proof given in [BE95, Theorem 1.2] can be adapted to the relative setting without problem.

Now we prove part (2). We consider the Leray spectral sequence $E_{2}^{i j}=H^{i}\left(R^{j} p_{*} \mathscr{M}\right)$, where $\mathscr{M}=\Omega_{\mathscr{Y} / U}^{*} \otimes \mathbb{E}$. The sequence abuts to $H^{l}(\mathscr{Y}, \mathscr{M})$ and gives the five-term exact sequence

$$
0 \longrightarrow H^{1}\left(p_{*} \mathscr{M}\right) \longrightarrow H^{1}(\mathscr{M}) \stackrel{\rho}{\longrightarrow} H^{0}\left(R^{1} p_{*} \mathscr{M}\right) \longrightarrow H^{2}\left(p_{*} \mathscr{M}\right) \longrightarrow H^{2}(\mathscr{M}) .
$$

Since $U$ is affine and $p$ is smooth, $\rho$ becomes an isomorphism

$$
\operatorname{Ext}_{\mathscr{Y}}^{1}\left(\Omega_{\mathscr{Y} / U}, \mathbb{E}\right) \stackrel{\sim}{\longrightarrow} H^{0}\left(R^{1} p_{*}\left(\Omega_{\mathscr{Y} / U}^{*} \otimes \mathbb{E}\right)\right) .
$$

Now we prove part (3). The morphism $\tilde{p}$ is proper because $p$ is and it is also flat because $p$ is and $\mathbb{E}$ is locally free. On the other hand, by (4.3.1), the restriction of an element $\varepsilon \in \operatorname{Ext}_{\mathscr{Y}}^{1}\left(\Omega_{\mathscr{Y}} / U, \mathbb{E}\right)$ to $\mathscr{Y}_{u}$ yields an element $\varepsilon_{u} \in H^{1}\left(\Omega_{\mathscr{Y}_{u}}^{*} \otimes \mathbb{E}_{u}\right)=\operatorname{Ext}^{1}\left(\Omega_{\mathscr{Y}}, \mathbb{E}_{u}\right)$. This implies that the fiber of $\widetilde{\mathscr{Y}}$ over $u$ is the rope on $\mathscr{Y}_{u}$, with conormal bundle $\mathbb{E}_{u}$ corresponding to $\varepsilon_{u}$.

Finally parts (4), (5) and (6) are relative versions of Theorem 2.2(2), (3) and (4). The proof given in [Gon06, Proposition 2.1] translates word for word to the relative setting.

Proposition 4.4. Let $a, b$ be integers, $a \geqslant b+2$. Let $\mathscr{E}^{\prime}=\mathscr{O}_{\mathbf{P}^{1}}(-a+1) \oplus \mathscr{O}_{\mathbf{P}^{1}}(-b-1)$ and let $\mathscr{E}=\mathscr{O}_{\mathbf{P}^{1}}(-a) \oplus \mathscr{O}_{\mathbf{P}^{1}}(-b)$. Let $\tilde{Y}$ be a rope on $Y=\mathbf{P}^{1}$ with conormal bundle $\mathscr{E}$.

(1) There exists a flat family $\widetilde{\mathscr{Y}}$ over a neighborhood $T$ of $0 \in \mathbf{A}^{1}$ such that $\widetilde{\mathscr{Y}}_{0}=\widetilde{Y}$ and $\widetilde{\mathscr{Y}}_{t}$ is a rope of conormal bundle $\mathscr{E}^{\prime}$ if $t \neq 0$.

(2) Let $N \geqslant \max \{4, a-1\}$. Let $Y \stackrel{i}{\hookrightarrow} \mathbf{P}^{N}$ be an embedding of $Y$ as a rational normal curve of degree $N$ in $\mathbf{P}^{N}$. Then there are embeddings $\widetilde{Y} \stackrel{\tilde{\imath}}{\hookrightarrow} \mathbf{P}^{N}$ of $\widetilde{Y}$ extending $i$. For such an embedding $\tilde{\imath}, T$ and the family $\widetilde{\mathscr{Y}}$ over $T$ described in part (1) can be chosen in such a way that $\tilde{\imath}$ extends to a family of embeddings $\widetilde{\mathscr{Y}} \hookrightarrow \mathbf{P}_{T}^{N}$. 


\section{F. J. Gallego, M. González and B. P. Purnaprajna}

Proof. It is well known (see for instance [Har81]) that $\mathscr{E}^{\prime}$ can be degenerated to $\mathscr{E}$. More precisely, consider the family $\mathscr{Y}=\mathbf{P}^{1} \times \mathbf{A}^{1} \stackrel{p_{1}}{\longrightarrow} \mathbf{A}^{1}$. There is a vector bundle $\mathbb{E}$ of rank 2 on $\mathscr{Y}$ such that the restriction of $\mathbb{E}$ to $\mathbf{P}^{1} \times\{0\}$ is $\mathscr{E}$ and the restriction $\mathbb{E}_{t}$ of $\mathbb{E}$ to $\mathbf{P}^{1} \times\{t\}$ is $\mathscr{E} \prime$ if $t \neq 0$. The rope $\widetilde{Y}$ corresponds to an element $\zeta \in \operatorname{Ext}^{1}\left(\Omega_{\mathbf{P}^{1}}, \mathscr{E}\right)=\mathrm{H}^{1}\left(\Omega_{\mathbf{P}^{1}}^{*} \otimes \mathscr{E}\right)$. A rope with conormal bundle $\mathscr{E}^{\prime}$ corresponds to an element of $\operatorname{Ext}^{1}\left(\Omega_{\mathbf{P}^{1}}, \mathscr{E}^{\prime}\right)=\mathrm{H}^{1}\left(\Omega_{\mathbf{P}^{1}}^{*} \otimes \mathscr{E}^{\prime}\right)$. Let $p_{2}$ be the projection of $\mathbf{P}^{1} \times \mathbf{A}^{1}$ onto $\mathbf{P}^{1}$. By Proposition 4.3 , a section of $R^{1} p_{1 *}\left(p_{2}^{*}\left(\Omega_{\mathbf{P}^{1}}^{*}\right) \otimes \mathbb{E}\right)$ yields a family $\widetilde{\mathscr{Y}}$, flat over $\mathbf{A}^{1}$, such

that $\widetilde{\mathscr{Y}_{0}}$ is a rope with conormal bundle $\mathbb{E}_{0}=\mathscr{E}$ and $\widetilde{\mathscr{Y}_{t}}$ is a rope with conormal bundle $\mathbb{E}_{t}=\mathscr{E}^{\prime}$ if $t \neq 0$. Since the fibers of $\mathscr{Y} \stackrel{p_{1}}{\longrightarrow} \mathbf{A}^{1}$ have dimension $1, R^{1} p_{1 *}\left(p_{2}^{*}\left(\Omega_{\mathbf{P}^{1}}^{*}\right) \otimes \mathbb{E}\right)(t)=H^{1}\left(\Omega_{\mathbf{P}^{1}}^{*} \otimes \mathbb{E}_{t}\right)$ for all $t \in \mathbf{A}^{1}$ (see [Mum70, II.5, Corollary 3]). Then we can lift $\zeta \in \mathrm{H}^{1}\left(\Omega_{\mathbf{P}^{1}}^{*} \otimes \mathscr{E}\right)$ to a section of the restriction of $R^{1} p_{1 *}\left(p_{2}^{*}\left(\Omega_{\mathbf{P}^{1}}^{*}\right) \otimes \mathbb{E}\right)$ to a suitable neighborhood $T$ of 0 . This proves part $(1)$.

Now we prove part (2). The existence of embeddings $\tilde{\imath}$ follows by Theorem 3.2. Let $\mathscr{I}$ be the ideal sheaf of $i(Y)$ in $\mathbf{P}^{N}$. The embedding $\tilde{\imath}$ corresponds to a surjective homomorphism $\tau_{0} \in$ $\operatorname{Hom}\left(\mathscr{I} / \mathscr{I}^{2}, \mathbb{E}_{0}\right)$. On the other hand, the conditions satisfied by $N$ imply that $h^{0}\left(\mathscr{I} / \mathscr{I}^{2 *} \otimes \mathbb{E}_{t}\right)$ is constant for all $t \in \mathbf{A}^{1}$. Then $\tau_{0}$ can be lifted to a local section $\tau$ of $p_{1 *}\left(p_{2}^{*}\left(\mathscr{I} / \mathscr{I}^{2^{*}}\right) \otimes \mathbb{E}\right)$. Since $p_{1}$ is proper, there is an open neighborhood $T$ of $0 \in \mathbf{A}^{1}$ such that $\tau$ is surjective on $p_{1}^{-1} T$. Then Proposition 4.3 implies part (2).

Now we are ready to prove the main theorem of this section.

Theorem 4.5. Let $\tilde{Y}$ be a rope on $Y=\mathbf{P}^{1}$ with arithmetic genus $g \geqslant 0$, Maroni invariant $n$ and conormal bundle $\mathscr{E}=\mathscr{O}_{\mathbf{P}^{1}}(-a) \oplus \mathscr{O}_{\mathbf{P}^{1}}(-b), a \geqslant b$. Let $N_{0}=\max \{4, a-1\}$ (i.e. $N_{0}=$ $\max \{4,(g+n) / 2\})$. Let $Y \stackrel{i}{\hookrightarrow} \mathbf{P}^{N} \subset \mathbf{P}^{M}$ be an embedding of $Y$ as a rational normal curve in $\mathbf{P}^{N}$, where $M=N+h^{0}\left(\mathscr{E} \otimes \mathscr{O}_{\mathbf{P}^{1}}(N)\right)$. If $N \geqslant N_{0}$, then the following occurs:

(1) there exist embeddings $\tilde{Y} \stackrel{\tilde{\imath}}{\hookrightarrow} \mathbf{P}^{N}$ extending $i$; for each such $\tilde{\imath}$, there exist an affine curve $T$ and a flat family $\widetilde{\mathscr{Y}}$ of subschemes of $\mathbf{P}^{N}$ such that $\widetilde{\mathscr{Y}}=\tilde{\imath}(\widetilde{Y})$ and $\widetilde{\mathscr{Y}} t$ is a smooth, irreducible curve if $t \neq 0$;

(2) there exist embeddings $\tilde{Y} \stackrel{\tilde{j}}{\hookrightarrow} \mathbf{P}^{M}$ extending $i$ such that $\tilde{j}$ embeds $\tilde{Y}$ as a linearly normal subscheme of $\mathbf{P}^{M}$; for each such $\tilde{\jmath}$, there exist an affine curve $T$ and a flat family $\widetilde{\mathscr{Y}}$ of subschemes of $\mathbf{P}^{M}$ such that $\widetilde{\mathscr{Y}}=\tilde{\jmath}(\widetilde{Y})$ and $\widetilde{\mathscr{Y}}_{t}$ is a smooth, irreducible curve if $t \neq 0$.

In particular, all ropes of multiplicity 3 on $\mathbf{P}^{1}$ with non-negative arithmetic genus are smoothable.

Proof. Case (1) We assume first that $n \leqslant(g+2) / 3$. This implies, together with Theorem 4.1, the existence of a smooth irreducible cover $X \stackrel{\pi}{\rightarrow} Y$ with $\pi_{*} \mathscr{O}_{X}=\mathscr{O}_{\mathbf{P}^{1}} \oplus \mathscr{E}$. On the other hand, the bound on $N$ in terms of $n$ and $g$ implies that $\mathscr{E} \otimes \mathscr{O}_{Y}(1)$ is non-special. Then the result follows from Theorems 2.4 and 3.2 and Corollary 3.6.

Case (2) We deal now with the remaining ropes. Proposition 4.4(1) yields that $\tilde{Y}$ sits into a flat family $\widetilde{\mathscr{Y}}$ over some affine curve $T$, whose other members are more balanced ropes $\widetilde{Y}^{\prime}$. More precisely, the ropes $\widetilde{Y}^{\prime}$ have all the same Maroni invariant $n-2$. Now, since $N \geqslant \max \{4, a-1\}$, by Proposition 4.4(2), there exists a family of embeddings $\widetilde{\mathscr{Y}} \hookrightarrow \mathbf{P}_{T}^{N}$. Arguing as in Corollary 3.6, we also have a family $\widetilde{\mathscr{Y}} \hookrightarrow \mathbf{P}_{T}^{M}$ of morphisms induced by complete linear series. Now let $\mathscr{H}$ be the Hilbert scheme of subschemes of $\mathbf{P}^{N}$ of dimension 1 , degree $3 N$ and arithmetic genus $g$. The Hilbert point of $\widetilde{Y}$ lies in the closure of the locus parametrizing ropes with Maroni invariant $n-2$, and, eventually, in the closure of ropes with Maroni invariant satisfying the conditions of case (1). Since the latter lie in the closure parametrizing smooth curves, so does $\widetilde{Y}$. Hence $\tilde{\imath}(\widetilde{Y}) \subset \mathbf{P}^{N}$ is smoothable. The same argument shows that $\tilde{\jmath}(\widetilde{Y}) \subset \mathbf{P}^{M}$ is smoothable.

We end by remarking explicitly, as we did in $\S 2$, that there are many different smoothings that we can exhibit for a given rope $\widetilde{Y}$. 


\section{DEFORMATION OF FINITE MORPHISMS AND SMOOTHING OF ROPES}

Remark 4.6. Given a rope $\tilde{Y}$ of multiplicity 3 on $\mathbf{P}^{1}$, there are many different smoothings of $\tilde{Y}$ obtained according to the results of this paper.

(1) As seen in $\S 3$, there are many different embeddings of $\widetilde{Y}$ in projective space.

(2) In the case where the conormal of $\tilde{Y}$ satisfies the conditions of Theorem 4.1, then there are many possible smooth covers $\pi$ to choose (see [Mir85]).

(3) Once $\pi$ is chosen and $\varphi$ is therefore fixed, the element $\tau \in \operatorname{Hom}\left(\mathscr{N}_{Y, Z}^{*}, \mathscr{E}\right)$ corresponding to $(\tilde{Y}, \tilde{\imath})$ may have, in most cases, many different liftings to $H^{0}\left(\mathscr{N}_{\varphi}\right)$. Liftings $\mu$ of $\tau$ to $H^{0}\left(\mathscr{N}_{\varphi}\right)$ correspond to first-order infinitesimal deformations $\widetilde{\varphi}$ of $\varphi$, such that $(\operatorname{im} \widetilde{\varphi})_{0}=\tilde{Y}$. For details of this see [Gon06, §3].

(4) There are many different ways of extending $\widetilde{\varphi}$ to a family of morphisms $\Phi$, as the proof of Theorem 1.1 shows.

\section{ACKNowledGements}

We thank N. Mohan Kumar for some valuable discussions and comments. We also thank E. Sernesi for some enlightening comments. The first two authors were partly supported by MEC grant BFM2003-03971 and by Complutense grant PR27/05-13876. They also thank the Department of Mathematics of the University of Kansas for its warm hospitality. The third author thanks the General Research Fund of Kansas for partly supporting this research project. He also thanks the Department of Algebra of the Universidad Complutense de Madrid for its warm hospitality.

\section{REFERENCES}

BE95 D. Bayer and D. Eisenbud, Ribbons and their canonical embeddings, Trans. Amer. Math. Soc. 347 (1995), 719-756.

EH83 D. Eisenbud and J. Harris, Divisors on general curves and cuspidal rational curves, Invent. Math. 74 (1983), 371-418.

Fon93 L. Y. Fong, Rational ribbons and deformation of hyperelliptic curves, J. Algebraic Geom. 2 (1993), 295-307.

GGP07 F. J. Gallego, M. González and B. P. Purnaprajna, Smoothing of double structures on Enriques surfaces, J. Pure Appl. Algebra (2007), doi:10.1016/j.jpaa.2007.07.021.

Gon06 M. González, Smoothing of ribbons over curves, J. reine angew. Math. 591 (2006), 201-235.

Gro61 A. Grothendieck, EGA III, Étude cohomologique des faisceaux cohérents (première partie), Publ. Math. Inst. Hautes. Études Sci. 11 (1961).

Gro62 A. Grothendieck, Technique de descente et théorèmes d'existence en géométrie algébrique V. Les schémas de Picard: Théorèmes d'existence, Séminaire Bourbaki, vol. 232, 1961-62.

Har81 J. Harris, A bound on the geometric genus of projective varieties, Ann. Scuola Norm. Sup. Pisa Cl. Sci. (4) 8 (1981), 35-68.

Mir85 R. Miranda, Triple covers in algebraic geometry, Amer. J. Math. 107 (1985), 1123-1158.

Mum70 D. Mumford, Abelian varieties, Tata Institute of Fundamental Research Studies in Mathematics, no. 5 (Oxford University Press, London, 1970).

Francisco Javier Gallego gallego@mat.ucm.es Departamento de Álgebra, Universidad Complutense de Madrid, Ciudad Universitaria, 28040 Madrid, Spain 


\section{DEFORMATION OF FINITE MORPHISMS AND SMOOTHING OF ROPES}

Miguel González mgonza@mat.ucm.es

Departamento de Álgebra, Universidad Complutense de Madrid, Ciudad Universitaria, 28040 Madrid, Spain

Bangere P. Purnaprajna purna@math.ku.edu

Department of Mathematics, University of Kansas, Snow Hall, 1460 Jayhawk Boulevard, Lawrence, KS 66045-7523, USA 